\title{
Clinical Outcome of Intra-articular Injection of Platelet-rich Plasma in Early-stage Knee Osteoarthritis in North Indian Patients
}

\author{
${ }^{1}$ Madhuresh Kumar, ${ }^{2}$ Santosh Kumar, ${ }^{3}$ Tulika Chandra, ${ }^{4}$ Vineet Sharma
}

\begin{abstract}
Introduction: With increasing frequency, platelet-rich plasma (PRP) preparations have been used to treat cartilage lesions to regenerate tissue homeostasis and retard the progression of knee osteoarthritis (OA).

Purpose: To determine the effectiveness of intra-articular PRP injections in early-stage OA patients and to evaluate the clinical outcome.

Materials and methods: This prospective study was conducted
in the Department of Orthopaedic Surgery, King George's
Medical University, Lucknow, Uttar Pradesh, India. The study
was conducted on patients with early-stage OA knee enrolled
on outpatient department basis during the year 2014 to 2015 .
The effective sample size was 40 patients with bilateral OA
knee in which intra-articular injection was given. And the clinical
outcomes and effectiveness were measured in terms of visual
analog scale and Western Ontario and McMaster Universities
Osteoarthritis Index scores at the end of 6,12 , and 24 weeks.
A p-value < 0.05 was considered statistically significant.
\end{abstract}

Results: There was significant improvement in all scores at the end of 6,12 , and 24 weeks.

Conclusions: The PRP treatment showed positive effects in patients with knee OA.

Clinical relevance: There are only a few studies of PRP treatment for cartilage on OA knees. Different PRP products might be more or less appropriate to treat different types of tissues and pathologies. The clinical efficacy of PRP remains under debate, and a standardized protocol has not yet been established.

Keywords: Intra-articular injection, Osteoarthritic knee, Plateletrich plasma.

How to cite this article: Kumar M, Kumar S, Chandra T, Sharma V. Clinical Outcome of Intra-articular Injection of Platelet-rich

\footnotetext{
${ }^{1}$ Senior Resident, ${ }^{2-4}$ Professor

1,4 Department of Orthopedics, King George's Medical University Lucknow, Uttar Pradesh, India

${ }^{2}$ Department of Orthopedic Surgery, King George's Medical University, Lucknow, Uttar Pradesh, India

${ }^{3}$ Department of Transfusion Medicine, King George's Medical University, Lucknow, Uttar Pradesh, India

Corresponding Author: Santosh Kumar, Professor, Department of Orthopedic Surgery, King George's Medical University Lucknow, Uttar Pradesh, India, Phone: +919415016349, e-mail: skkgmu69@gmail.com
}

Plasma in Early-stage Knee Osteoarthritis in North Indian Patients. J Postgrad Med Edu Res 2018;52(2):56-61.

Source of support: Nil

Conflict of interest: None

\section{INTRODUCTION}

Osteoarthritis is a chronic degenerative joint disease affecting millions of people worldwide. ${ }^{1}$ The disease is a leading cause of disability in the elderly, causing pain, stiffness, and loss of function in articulating joints. Osteoarthritis is characterized by changes in the anatomy of load-bearing joints that lead to degradation of articular cartilage, inflammation of the synovium (synovitis), changes to subchondral bone, and growth of new bone and cartilage (osteophytes) at the joint edge., ${ }^{2,3}$

Various treatment modalities have been described for OA, including medical ones like nonsteroidal antiinflammatory drugs (NSAIDs), corticosteroids, viscosupplementation, and surgical modalities like high tibial osteotomies and knee joint replacements for the knee. ${ }^{4}$ Apart from weight reduction and avoiding activities that exert excessive stress on the joint cartilage, there has been no specific treatment to prevent cartilage degeneration or to repair damaged cartilage in OA. Therefore, for the past several years, research has focused on determining the causes of knee OA and to discover how to stop the progression of the disease, aside from lowering the effects, such as pain and discomfort by therapy. ${ }^{5}$

Recently, PRP is emerging as a therapeutic alternative as cited in various studies. ${ }^{6-9}$ The use of PRP seems to be a suitable alternative in North Indian population, at least in the initial stages because most of the patients come to us from far-flung poverty-stricken areas and are not always ready for replacement. So, it is imperative to prevent OA from progressing to a higher stage by the help of PRP injection and the use of physical therapies at the initial stages.

Platelet-rich plasma has been classically described as "a volume of plasma that has a platelet count above baseline." The PRP is prepared by withdrawing peripheral blood and by centrifugation to obtain a highly concentrated sample of platelets. The platelets undergo degranulation to release growth factors with healing properties. The plasma 
contains cytokines, thrombin, and other growth factors, with inherent biological and adhesive properties. ${ }^{10}$

The present prospective study was conducted at King George's Medical University, Lucknow, from 2013 to 2015 on 40 patients with bilateral OA of knee in order to assess the role of single intra-articular PRP injection prepared from the patient's own blood.

\section{MATERIALS AND METHODS}

This study was a prospective study of 40 patients with bilateral knee OA (based on American College of Rheumatology criteria) who were all followed up for a minimum period of 1 year. Patients between 45 and 70 years of age with Kellgren and Lawrence (KL) grade ${ }^{11}$ 0 to 3 having painful knees for more than 6 months and giving informed consent to participate in the study were enrolled. Patients who did not wish to participate in the study and those having some contraindication to PRP injection like thrombocytopenia, anticoagulant therapy, active infections in or around the knee joint, presence of any primary or secondary malignancy, and pregnancy were excluded.

All the patients underwent a common protocol of management. After proper informed consent and history taking, clinico-radiological assessment was done. The KL grading of knee OA was done based on X-rays. We included patients with OA of KL grades 1 to 3 . Grade IV patients were excluded. Severity of symptoms was assessed by visual analog scale (VAS)/Western Ontario and McMaster Universities Osteoarthritis Index (WOMAC) score (version VA 3.1). ${ }^{12,13}$ The patients were explained about the procedure and the risks associated with it. Physical therapy consisting of quadriceps exercises was instructed to the patients. For the preparation of PRP, $60 \mathrm{~mL}$ of peripheral blood was collected by the transfusion medicine department of our university maintaining strict sterility protocol. The collected blood underwent a series of centrifugation process, thereby delivering the desired PRP with four- to six-fold increase in platelet concentration. To ensure the increase in the platelet count, hematological analysis was done for the peripheral blood and the final PRP. Intra-articular administration of PRP was done by injecting $4 \mathrm{~mL}$ of platelet concentrate in the suprapatellar pouch through superolateral approach with a 21-gauge needle. No form of local anesthetic was used. Immediately after the injection, passive flexion and extension of the affected knee were performed three times. The patients were observed for 30 minutes, following which they were given acetaminophen for pain on "sos basis" and prophylactic oral antibiotics for 3 days. They were instructed to limit the use of their affected knee for 24 hours. The patients were especially instructed not to use any NSAIDs. (Paracetamol was used as a rescue medication.) They were asked to stop medications 48 hours before the follow-up assessment.

The patients were evaluated for subjective improvement in pain, stiffness, and physical function by the WOMAC score at 6 weekly intervals for 1 year.

\section{OBSERVATION AND RESULTS}

Out of 40 patients who were available for final followup at 1 year, females outnumbered the males in a ratio of 1.86:1. Most of the patients belonged to 50 to 60 years $(n=23)$ followed by the age group $>60$ years $(n=10)$. Most of the patients were in K-L grade III (Table 1). A reduction in WOMAC scores was evident from the first follow-up at 6 weeks itself. All the patients had maximum relief (least WOMAC) at 6 weeks' follow-up (Table 2 and Graph 1$)$. Even at 1-year follow-up, many patients $(n=27)$ had a lesser mean WOMAC score than the baseline.

If we look at the individual components of WOMAC, maximum change was seen in the WOMAC pain subscale, followed by the physical function subscale. The stiffness subscale showed the least improvement. The change in the WOMAC (pain) subscale was highly significant (p-value <0.001) up to 6 months. The WOMAC (physical function) accorded change, to somewhat lesser extent, p-value $<0.001$ at 6 and 12 weeks, and $<0.05$ at 24 weeks. The WOMAC (stiffness) was significantly different at 6 and 12 weeks, following which the p-value became

Table 1: Association between various patient-related factors (sex, age, $\mathrm{KL}$ grade) and difference in WOMAC (total) scores at baseline and at 6 weeks' follow-up

\begin{tabular}{lllllll}
\hline Parameters & & $\begin{array}{l}\text { No. of } \\
\text { patients }\end{array}$ & $\begin{array}{l}\text { WOMAC (total) } \\
\text { at baseline }(A)\end{array}$ & $\begin{array}{l}\text { WOMAC (total) } \\
\text { at 6 weeks }(B)\end{array}$ & $\begin{array}{l}\text { Difference in scores at baseline } \\
\text { and at 6 weeks }(A-B)\end{array}$ & $p$-value \\
\hline Sex & Males & 14 & $59.14 \pm 6.837$ & $24.36 \pm 3.249$ & $34.79 \pm 5.754$ & 0.4004 \\
& Females & 26 & $60.08 \pm 7.746$ & $27.46 \pm 11.046$ & $32.85 \pm 7.401$ & 0.0557 \\
Age & 40 to $<50$ years & 7 & $59.29 \pm 8.440$ & $20.71 \pm 4.071$ & $38.57 \pm 6.214$ & 0.0912 \\
& 50 to $<60$ years & 23 & $57.00 \pm 6.135$ & $23.87 \pm 4.060$ & $33.13 \pm 5.111$ & $30.30 \pm 9.889$ \\
& $\geq 60$ years & 10 & $66.40 \pm 5.190$ & $36.10 \pm 12.905$ & $23.00 \pm 7.071$ & $33.25 \pm 6.299$ \\
KL grade & 1 & 2 & $44.50 \pm 0.707$ & $21.50 \pm 6.364$ & \\
& 2 & 24 & $56.167 \pm 6.592$ & $22.92 \pm 4.942$ & \\
& 3 & 26 & $63.46 \pm 5.573$ & $28.85 \pm 10.291$ & $34.62 \pm 7.788$ & \\
\hline
\end{tabular}


Table 2: WOMAC and VAS scores at the time of initial visit (baseline) and at sequential follow-ups

\begin{tabular}{|c|c|c|c|c|c|c|c|c|}
\hline \multirow[b]{2}{*}{ Visits } & \multicolumn{2}{|c|}{ WOMAC (pain) } & \multicolumn{2}{|c|}{ WOMAC (stiffness) } & \multicolumn{2}{|c|}{ WOMAC (physical function) } & \multicolumn{2}{|c|}{ VAS } \\
\hline & Mean $\pm S D$ & $p$-value & Mean $\pm S D$ & $p$-value & Mean $\pm S D$ & $p$-value & Mean $\pm S D$ & $p$-value \\
\hline Baseline & $14.23 \pm 1.874$ & - & $4.38 \pm 0.807$ & - & $41.15 \pm 5.182$ & - & $13.70 \pm 2.139$ & - \\
\hline 6 weeks & $5.80 \pm 2.301$ & $<0.001$ & $3.25 \pm 0.707$ & $<0.001$ & $17.33 \pm 6.818$ & $<0.001$ & $6.202 \pm 2.812$ & $<0.001$ \\
\hline 12 weeks & $8.20 \pm 2.462$ & $<0.001$ & $3.58 \pm 0.747$ & $<0.001$ & $23.70 \pm 7.003$ & $<0.001$ & $7.655 \pm 2.815$ & $<0.001$ \\
\hline 24 weeks & $10.83 \pm 2.395$ & $<0.001$ & $3.95 \pm 1.037$ & $>0.05$ & $37.20 \pm 5.727$ & $<0.05$ & $9.93 \pm 3.261$ & $<0.001$ \\
\hline 52 weeks & $13.20 \pm 2.301$ & $>0.05$ & $4.48 \pm 0.96$ & $>0.05$ & $40.58 \pm 6.147$ & $>0.05$ & $12.63 \pm 2.488$ & $>0.05$ \\
\hline
\end{tabular}
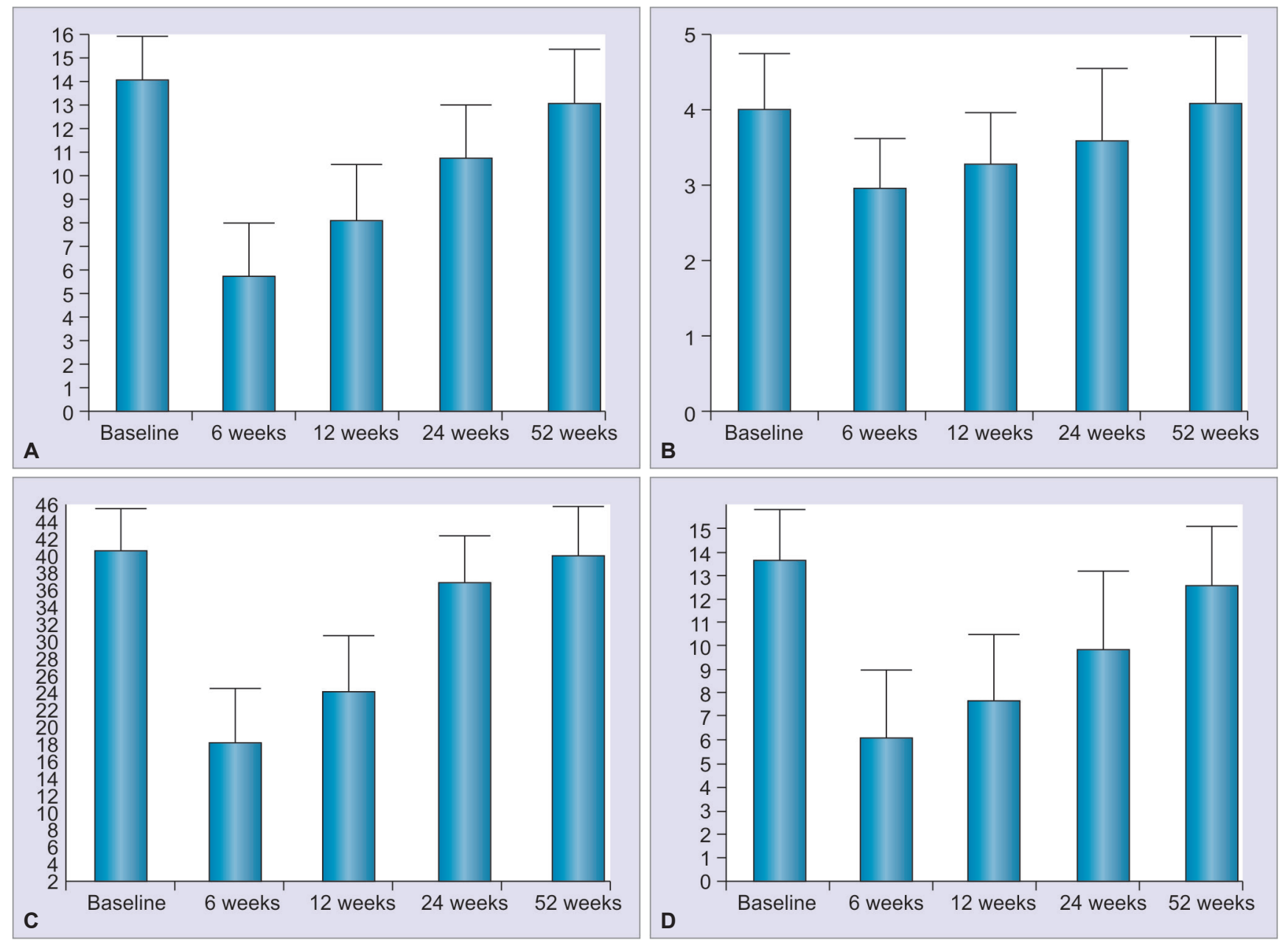

Graphs 1A to D: Error-bar plot of WOMAC scores. (A) Pain, (B) stiffness, (C) physical function, and (D) VAS

$>0.05$. The change in VAS was similar to the WOMAC (pain) subscale.

We also tried to find out if the change in the WOMAC (total) scores was significantly different as regards age (40-50, 50-60, >60), sex (males vs females), and KL grading (grades 1 or 2 or 3 ) of the patient. For this, we have compared the values at baseline with that at 6 weeks. We could not find any statistically significant difference in any of the three parameters. However, clinically, we found a greater change in lesser age groups $(38.57 \pm 6.214$, in $40-<50$ years patients), (33.13 \pm 5.111 , in $50-<60$ years patients) as compared with the elderly ones (30.30 \pm 9.889 , in $\geq 60$ years). However, it was not statistically significant $(\mathrm{p}$-value $=0.0557)($ Table 1$)$.
Statistical analysis was done by GraphPad InStat software, San Diego, USA. First of all, it was ensured that the individual data of WOMAC scores and VAS (pain) followed Gaussian distribution. Thereafter, the changing trend of all the scores was summarized in the form of means and standard deviation (SD), following which, one-way analysis of variance (ANOVA) using Tukey-Kramer multiple comparison test was done. To compare the change in the WOMAC scores at 6 weeks from baseline in terms of age, sex, and KL grade, either Student's t-test (where there were two groups) or ANOVA (in cases of three or more groups) was used to calculate the $\mathrm{p}$-value and to see if the difference was significant. 
Among the complications, self-limiting injection site pain was present in $27.5 \%$ of cases $(n=11)$, which improved within a maximum of 3 days. Few patients $(n=5)$ complained of transient knee swelling and stiffness after the intra-articular injections. No patient complained of any features suggestive of infection. No case of prolonged bleeding was noted.

\section{DISCUSSION}

This study has reemphasized the fact that intra-articular injection of PRP causes subjective improvements in the OA patients.

The PRP exerts multiple biological actions, including modulatory effects on inflammation and angiogenesis, which may translate clinically to pain relief. ${ }^{14}$

The fact that PRP can cause relief for almost a year or even beyond highlights the fact that the growth factors released from the platelets prevent OA progression and causes intra-articular changes. Whether PRP causes healing of hyaline articular cartilage is a matter of debate. In isolated chondral lesions, healing has been seen as described in some studies. ${ }^{15,16}$ But till now, we have not achieved any significant evidence of healing of articular cartilage in OA. Hassan et $\mathrm{al}^{17}$ evaluated the osteoarthritic knees before and after the PRP injections and have found a significant decrease in the number of patients having increased Doppler activity after 6 PRP injections. Also, there was a highly significant decrease in the number of patients having synovial hypertrophy. Sampson et $\mathrm{al}^{9}{ }^{9}$ in a pilot study on 12 patients, tried to quantify the intra-articular changes in terms of ultrasonic assessment of femoral condyle cartilage thickness, a method previously demonstrated as reproducible. With the knee in flexion, the cartilage thickness was measured at the lateral condyle, medial condyle, and intercondylar notch. Measurements were taken at the preinjection and 6-month follow-up visits. Although there was some thickening in the lateral and central locations, none of the post minus pre changes were statistically significant. In a recent study, Halpern et al ${ }^{8}$ showed that qualitative Magnetic resonance imaging (MRI) demonstrated no change per compartment in at least $73 \%$ of cases at 1 year. We feel that a more objective evaluation in terms of cartilage thickness by quantitative MRI in a long-term study needs to be done to address the issue.

In our study, we have tried to see the effect of a single-shot injection up to 1 year. Various studies, ${ }^{9,18,19}$ which have compared PRP and HA, have resorted to three injections at monthly intervals, the regimen commonly followed for HA. Some of the published literature has resorted to monthly injections twice. ${ }^{20}$ Few papers describe the use of weekly injections thrice. ${ }^{21,22}$ No consensus has been drawn till date about the frequency and dosing intervals of PRP injections. Few recent studies have highlighted better pain relief with multiple injections. ${ }^{23}$ Proper randomized control trials comparing the different dosing patterns are required.

In this study, we have used PRP with a count between 400 and $600 \%$ from the baseline, which in all the cases was confirmed hematologically. The platelet quality influences effectiveness, including platelet content, leukocytes, and growth factor concentration, because preparations of PRP have no selection criterion. The platelet count in PRP may vary from two- to several-fold, depending on the donor's physical condition, age, or gender, which leads to unstable and nonrepeatable PRP treatment. ${ }^{10}$ So, the results may vary from one preparation to the other. Proper standardization should be done to compare various studies and formulate recommendations.

The study was done on North Indian patients, where due to the traditional and religious concerns, squatting and sitting cross-legged is a part of daily lifestyle, both of which favor OA of knee. Many patients, especially females, do present to us early (KL grades 1-3) with pain in single or both knee joints. So, we have got a good disease burden which can be subjected to PRP injection.

In this study, the most consistent effect was pain relief, followed by physical function. Improvement in stiffness was seen to a somewhat lesser extent, though statistically significant. Based on laboratory studies, PRP is said to have numerous proteins which can have an analgesic effect. Other modalities of management of OA like NSAIDs cause only short-term pain relief. But the analgesia caused by PRP is prolonged and sustained, at least for 6 months. The analgesic effect seems to wane off over the next few months as seen in numerous other studies as well. Whether this effect is due to only biochemical alterations within the joint or structural changes as well needs to be evaluated. Above all, among the plethora of growth factors present in PRP, not all the molecules are responsible for the desired effect. Some of the growth factors present in PRP, such as TGF-beta and bFGF have negative effects on the OA joint which differ from the effects in more normal joints. ${ }^{10}$ Metcalf et $\mathrm{al}^{24}$ also feel that although the basic scientific rationale surrounding PRP products is compelling, the clinical application has outpaced the research. Probably, more new research studies in the field of basic sciences will help us find the answer.

In our study, we found age to be an important factor determining the clinical outcome (clinically significant, but not statistically). As OA is an age-related degenerative process, it may happen that PRP is more beneficial at incipient stages as compared with the later, when the disease has already progressed. It can also happen that other factors like physical strength of the muscles, which gradually wanes with age, may be a confounding factor. 
This observation of ours goes well with other studies.

This method appears to be quite safe in view of the fact that it is an autologous preparation, and hence chances of any immunological or allergic reactions are theoretically nil. We have experienced the same in our study wherein the most significant complication that we encountered was injection site pain and transient fullness of the joint. The same has been noted by Sánchez et $\mathrm{al}^{25}$ and other studies. ${ }^{26}$ However, the risks associated with any intraarticular injections in the form of infection, bleeding, bruising, peripheral nerve injury, allergy to local anesthetics, and temporary exacerbation of stiffness and soreness cannot be ruled out. Proper care like sterile precautions, evaluation for the presence of coagulation abnormalities and hypersensitivity to local anesthetics (if used, which was not used in our study) must be done.

Nevertheless, there were some limitations of our study. Firstly, we had no control group. Secondly, we did not compare the effect of single vs multiple injections of PRP at some fixed intervals. Thirdly, no form of objective assessment of intra-articular changes, either radiological or biochemical, was done to have a clinicopathological correlation.

\section{CONCLUSION}

Based on our study, we can say that intra-articular injection of autologous PRP is a safe, cheap, easy to prepare and use, and has a therapeutic role in early knee OA. But, several issues, such as standardization of PRP preparation, creation of a standard protocol, and evidence in favor of hyaline articular cartilage regeneration, if any, by quantitative MRI or some other objective method still remain to be looked into.

\section{ACKNOWLEDGMENTS}

The authors would like to thank the help of the Department of Transfusion Medicine, King George Medical University, Lucknow, Uttar Pradesh, India for the preparation of PRP.

\section{REFERENCES}

1. Wieland HA, Michaelis M, Kirschbaum BJ, Rudolphi KA. Osteoarthritis—an untreatable disease? Nat Rev Drug Discov 2005 Apr;4(4):331-344.

2. Goldring MB, Goldring SR. Articular cartilage and subchondral bone in the pathogenesis of osteoarthritis. Ann NY Acad Sci 2010 Mar;1192:230-237.

3. Felson DT. Developments in the clinical understanding of osteoarthritis. Arthritis Res Ther 2009 Jan;11(1):203.

4. Anandacoomarasamy A, March L. Current evidence for osteoarthritis treatments. Ther Adv Musculoskelet Dis 2010 Feb;2(1):17-28.

5. Bhatia D, Bejarano T, Novo M. Current interventions in the management of knee osteoarthritis. J Pharm Bioallied Sci 2013 Jan;5(1):30-38.
6. Filardo G, Kon E, Buda R, Timoncini A, Di Martino A, Cenacchi A, Fornasari PM, Giannini S, Marcacci M. Platelet-rich plasma intra-articular knee injections for the treatment of degenerative cartilage lesions and osteoarthritis. Knee Surg Sports Traumatol Arthrosc 2011 Apr;19(4):528-535.

7. Patel S, Dhillon MS, Aggarwal S, Marwaha N, Jain A. Treatment with platelet-rich plasma is more effective than placebo for knee osteoarthritis: a prospective, double-blind, randomized trial. Am J Sports Med 2013 Feb;41(2):356-364.

8. Halpern B, Chaudhury S, Rodeo AS, Hayter CL, Bogner E, Potter HG, Nguyen J. Clinical and MRI outcomes after platelet-rich plasma treatment for knee osteoarthritis. Clin J Sport Med 2013 May;23(3):238-239.

9. Sampson S, Reed M, Silvers H, Meng M, Mandelbaum B. Injection of platelet-rich plasma in patients with primary and secondary knee osteoarthritis: a pilot study. Am J Phys Med Rehabil 2010 Dec;89(12):961-969.

10. Zhu Y, Yuan M, Meng HY, Wang AY, Guo QY, Wang Y, Peng J. Basic science and clinical application of platelet-rich plasma for cartilage defects and osteoarthritis: a review. Osteoarthritis Cartilage 2013 Nov;21(11):1627-1637.

11. Kellgren JH, Lawrence JS. Radiological assessment of osteoarthrosis. Ann Rheum Dis 1957 Dec;16(4):494-502.

12. Bellamy, N. Womac osteoarthritis index. 2012. [cited 2012 Feb 21]. Available from: http://www.auscan.org/womac/index.htm.

13. Nicholas, B. WOMAC osteoarthritis index VA 3.1 user guide 5 . Australia: University of Queensland Faculty of Health Sciences; 2002. pp. 15-16.

14. Sánchez M, Guadilla J, Fiz N, Andia I. Ultrasound-guided platelet-rich plasma injections for the treatment of osteoarthritis of the hip. Rheumatology (Oxford) 2012 Jan;51(1):144-150.

15. Giannini S, Buda R, Battaglia M, Cavallo M, Ruffilli A, Ramponi L, Pagliazzi G, Vannini F. One-step repair in talar osteochondral lesions: 4-year clinical results and t2-mapping capability in outcome prediction. Am J Sports Med 2013 Mar;41(3):511-518.

16. Battaglia M, Rimondi E, Monti C, Guaraldi F, Sant'Andrea A, Buda R, Cavallo M, Giannini S, Vannini F. Validity of T2 mapping in characterization of the regeneration tissue by bone marrow derived cell transplantation in osteochondral lesions of the ankle. Eur J Radiol 2011 Nov;80(2):e132-e139.

17. Hassan AS, El-Shafey AM, Ahmed HS, Hamed MS. Effectiveness of the intra-articular injection of platelet rich plasma in the treatment of patients with primary knee osteoarthritis. Egypt Rheumatol 2015 Jul;37(3):119-124.

18. Spaková T1, Rosocha J, Lacko M, Harvanová D, Gharaibeh A. Treatment of knee joint osteoarthritis with autologous plateletrich plasma in comparison with hyaluronic acid. Am J Phys Med Rehabil 2012 May;91(5):411-417.

19. Raeissadat SA, Rayegani SM, Hassanabadi H, Fathi M, Ghorbani E, Babaee M, Azma K. Knee osteoarthritis injection choices: platelet-rich plasma (PRP) versus hyaluronic acid (A one-year randomized clinical trial). Clin Med Insights Arthritis Musculoskelet Disord 2015 Jan;8:1-8.

20. Gobbi A, Karnatzikos G, Mahajan V, Malchira S. Platelet-rich plasma treatment in symptomatic patients with knee osteoarthritis: preliminary results in a group of active patients. Sports Health 2012 Mar;4(2):162-172.

21. Ilhanli I, Guder N, Avci E. Is platelet-rich plasma a promising treatment in severe knee osteoarthritis? Unif J Med Med Sci 2015 Aug;1(1):001-005. 
22. Filardo G, Kon E, Di Martino A, Di Matteo B, Merli ML, Cenacchi A, Fornasari PM, Marcacci M. Platelet-rich plasma vs hyaluronic acid to treat knee degenerative pathology: study design and preliminary results of a randomized controlled trial. BMC Musculoskelet Disord 2012 Nov; 13:229.

23. Görmeli G1, Görmeli CA, Ataoglu B, Çolak C, Aslantürk O, Ertem K. Multiple PRP injections are more effective than single injections and hyaluronic acid in knees with early osteoarthritis: a randomized, double-blind, placebo-controlled trial. Knee Surg Sports Traumatol Arthrosc 2017 Mar;25(3): 958-965.
24. Metcalf KB, Mandelbaum BR, McIlwraith CW. Application of platelet-rich plasma to disorders of the knee joint. Cartilage 2013 Oct;4(4):295-312.

25. Sánchez M, Anitua E, Azofra J, Aguirre JJ, Andia I. Intraarticular injection of an autologous preparation rich in growth factors for the treatment of knee OA: a retrospective cohort study. Clin Exp Rheumatol 2008 Sep-Oct;26(5):910-913.

26. RayeganiSM, RaeissadatSA, TaheriMS, Babaee M, BahramiMH, Eliaspour D, Ghorbani E. Does intra articular platelet rich plasma injection improve function, pain and quality of life in patients with osteoarthritis of the knee? A randomized clinical trial. Orthop Rev (Pavia) 2014 Sep;6(3):5405. 